\title{
O USO DE FERRAMENTAS DA QUALIDADE PARA ANÁLISE DE MANIFESTAÇÕES PATOLÓGICAS POR BAIRROS NA CIDADE DE QUIXADÁ CEARÁ
}

\author{
MELO, SIZY BRENDA \\ Discente do curso de Engenharia de Produção Civil \\ IFCE \\ Ceará; Brasil \\ sizy.ifce@gmail.com
}

\author{
XAVIER, TÁLISSON DAVI NOBERTO \\ Docente do curso de Engenharia de Produção Civil \\ IFCE \\ Ceará; Brasil \\ talisson.xavier@ifce.edu.br
}

\author{
PEREIRA, THAÍS MARILANE CARNEIRO DE VASCONCELOS \\ Docente do curso de Engenharia de Produção Civil \\ IFCE \\ Ceará; Brasil \\ thais.pereira@ifce.edu.br
}

\section{RESUMO}

Novas ferramentas e tecnologias vêm sendo implementadas no setor da construção civil objetivando melhorar a qualidade, segurança e estética das edificações, além de prevenir o surgimento de manifestações patológicas. Apesar desses esforços, ainda é frequente a observação dessas manifestações, compreendidas como as anomalias nas construções, sendo necessária sua identificação e tratamento adequado. As ferramentas da qualidade são um conjunto de técnicas estatísticas básicas que auxiliam os processos de coleta, tratamento e disposição dos dados, auxiliando na tomada de decisão e direcionando os esforços de melhoria. O presente estudo teve como objetivo a identificação e análise das manifestações patológicas evidentes em residências de diferentes bairros da cidade de Quixadá - CE. Inicialmente foram escolhidos os bairros seguindo o critério de propensão para moradia, em seguida foram realizadas visitas in loco para registro fotográfico das anomalias, coleta e análise dos dados. Coletou-se os dados através da folha de verificação, em seguida tratou os mesmos com a curva ABC e a matriz GUT, elaborada com o auxílio do método Delphi e por fim, as possíveis causas foram dispostas por meio do diagrama de ishikawa. Os resultados demonstraram que aproximadamente $60 \%$ das anomalias encontradas eram referentes a apenas três manifestações patológicas: fissuras, descascamentos de pintura e desagregamento da argamassa. Quanto a ordem de priorização, as rachaduras foram apontadas como maior problema devido ao risco de comprometimento ao sistema estrutural, por isso foram investigadas suas causas e medidas preventivas foram propostas.

Palavras-chave: manifestações patológicas, curva ABC, matriz GUT, diagrama de causa e efeito.

\begin{abstract}
New tools and technologies are being implemented in the construction sector aiming at improving the quality, safety and aesthetics of buildings, and prevent the emergence of pathologies. Despite these efforts, It is still common to observe pathological manifestations, understood as the anomalies in the constructions, being required to identify and treat them properly. Quality tools are a combination of statistical techniques that assist the processes of data collection, processing and disposal, assisting in decision making and directing improvement efforts. The present study aimed to identify and analyze the pathologies in residences in different neighborhoods of the city of Quixadá, in northeastern Brazil. Initially, the city's neighborhoods were chosen. following the propensity criterion for housing, then on site visits were made for photograph anomalies, data collection and analysis. Data were collected through the check sheet, then treated same with the ABC curve and the G.U.T priority matrix and finally the possible causes were showed using ishikawa diagram. The results showed that approximately $60 \%$ of the anomalies found were related to only three pathologies: cracks, paint peeling and grout breakdown. Regarding the order of prioritization, the Cracks were identified as a major problem due to the risk of compromise to the structural system, so its causes were investigated and preventive measures were proposed. Keywords: pathological manifestations, ABC curve, GUT priority matrix, cause and effect diagram.
\end{abstract}




\section{INTRODUÇÃO}

Desde os nossos antepassados, a civilização busca encontrar recursos capazes de favorecer o suprimento de suas necessidades. Nessa pespectiva, a Indústria da Construção Civil (ICC) vêm tentando promover o bem estar da sociedade através do desenvolvimento de diversas obras de engenharia, visto que nos últimos anos, como ressalta Moreira e Marchini (2018), tem-se aumentado a demanda por construções em segmentos de infraestrutura e edificações.

Dada a sua importância, novas ferramentas e tecnologias vêm sendo implementadas no processo construtivo, objetivando melhorar a qualidade, segurança e estética das edificações, além de prevenir o surgimento de manifestações patológicas, proporcionando maior estabilidade e funcionalidade às moradias. Mas, apesar desses esforços, ainda é frequente a observação dessas anomalias nas construções que, para Vieira (2016), podem vir a comprometer a vida útil de uma edificação, sendo necessário o reconhecimento e tratamento adequado. Assim, faz-se válido as palavras de Souza e Ripper (1998), Figueiredo e Lapa (2008) e Santos e Soares (2014), no qual destacam a importância de se analisar as origens, manifestações e causas desses problemas.

Nesse sentido, já que algumas anomalias podem ser facilmente percebidas por meio da observação, as ferramentas da qualidade que, para Werkema (1995), são um conjunto de técnicas estatísticas básicas, podem auxiliar na coleta, tratamento e disposição dos dados, facilitando a identificação dos problemas, as tomadas de decisões e o direcionamento dos esforços de melhoria. Como ferramentas podem-se citar: Folha de Verificação, Curva ABC, Matriz GUT: Gravidade, Urgência e Tendência, e Diagrama de Ishikawa, que foram utilizadas para o desenvolvimento do presente trabalho.

Apresenta-se, portanto, essa pesquisa com o objetivo de identificar e analisar as manifestações patológicas baseando-se na triagem das anomalias mais recorrentes em residências dos bairros Centro, Alto São Francisco, Baviera e Lagoa da cidade de Quixadá - CE, por meio da aplicação das ferramentas da qualidade. Além disso, busca-se também, apontar a ordem para priorização de solução das anomalias, bem como fundamentando-se em bibliografias consultadas e estudadas identificar as possíveis causas e propor medidas mitigatórias para evitar problemas semelhantes.

\section{REFERENCIAL TEÓRICO}

\subsection{Patologia das construções}

Para Vieira (2016), as edificações podem ser comparadas aos seres humanos, já que assim como eles, estão sujeitas a acidentes e deterioração ao longo do tempo. Nesse sentido, surgem-se as manifestações patológicas que devem ser estudadas e analisadas. Deste modo, patologia, na cnstrução civil, refere-se a ciência que estuda as origens, formas de manifestações e impactos desses problemas nas construções (SOUZA, RIPPER, 1998). Para Santos e Soares (2014), Vieira (2016) e dentre outros, as manifestações patológicas podem ocorrer devido a falhas de projeto, execução e manutenção.

As falha nos projetos são provenientes, em muitos casos, de estudos preliminares deficientes e anteprojetos equivocados (SANTOS, SOARES, 2014). Assim, faz-se necessário seguir as Normas Técnicas que apontam diretrizes capazes de nortear o processo construtivo e garantir funcionalidade, segurança e durabilidade da estrutura, como ressalta Vieira (2016). Para Souza e Ripper (1998), na fase de execução, os problemas podem ser influenciados pela ausência de controle de qualidade, implementação de técnicas construtivas inadequadas, mão de obra desqualificada, materiais com baixa qualidade, bem como o não seguimento das especificações exigidas no projeto e memorial descritivo, enquanto que, na etapa de manutenção, as falhas advém da ausência de providências para conservação. Diante desse conjunto de fatores, surgem-se as fissuras, trincas, rachaduras, descascamentos de pintura, gretamento e outros problemas, sendo necessário, como ressalta Figueiredo e Lapa (2008), um adequado reparo, recuperação ou reforço.

\subsection{Ferramentas da qualidade}

As ferramentas da qualidade, para Werkema (1995), são um conjunto de técnicas estatísticas básicas que vêm ganhando destaque no mercado por auxiliar no processo de descrição e interpretação das variabilidades, ou seja, das causas que ocasionam defeitos em produtos ou em outros tipos de patrimônios. À vista disso, esses métodos podem auxiliar nos processos de coleta, tratamento e disposição dos dados, norteando a tomada de decisões e o direcionamento dos esforços de melhoria. Ressalta-se que são sete as ferramentas da qualidade: diagrama de causa e efeito, histograma, gráfico de 
pareto, diagrama de correlação, gráfico de controle e folha de verificação (WERKEMA, 1995). Mas, para fins deste trabalho, será utilizado apenas duas, no qual pode-se citar: folha de verificação e diagrama de causa e efeito, melhor descrito no subtópicos 2.2.1 e 2.2.4, além dessas, será aplicado outras duas que se derivaram das já existentes, curva ABC e Matriz GUT.

\subsubsection{Folha de verificação}

A folha de verificação, consiste em uma ferramenta que auxilia na coleta e análise dos dados, organizando-os em forma de tabelas ou planilhas (WERKEMA, 1995). Nota-se, que a mesma agrega valor ao processo de registro das informações devido a otimização do tempo, além de evitar retrabalhos e conclusões distorcidas, focando em parâmetros específicos por fazer uso da estratificação, que para Marshall Junior (2008), trata-se do desdobramento dos dados em grupos ou categorias.

\subsubsection{Curva ABC}

A curva $\mathrm{ABC}$, apesar de não ser uma das sete ferramentas da qualidade, pode ser compreendida como uma derivação do diagrama de pareto. Segundo Nobre, Melo e Matos (2019), é um método de classificação útil na identificação dos itens que necessitam de maior controle. Além disso, Solano e Paladini (2003), afirmam que essa filosofia facilita a identificação de problemas mais evidentes em termos quantitativos, o que favorece a tomada de decisões.

O percentual adotado para caracterização das classes ABC costuma ser de $20 \%, 30 \%$ e $50 \%$, respectivamente (DIAS, 1993 apud SIMÕES, RIBEIRO). No entando, vale ressaltar que esses percentuais podem sofrer alterações para uma melhor adaptação ao problema estudado e analisado. Com os dados ordenados plota-se o gráfico, onde no eixo das abcissas encontra-se quantidades de itens e no eixo das ordenadas o consumo acumulado, como mostra a Figura 1.

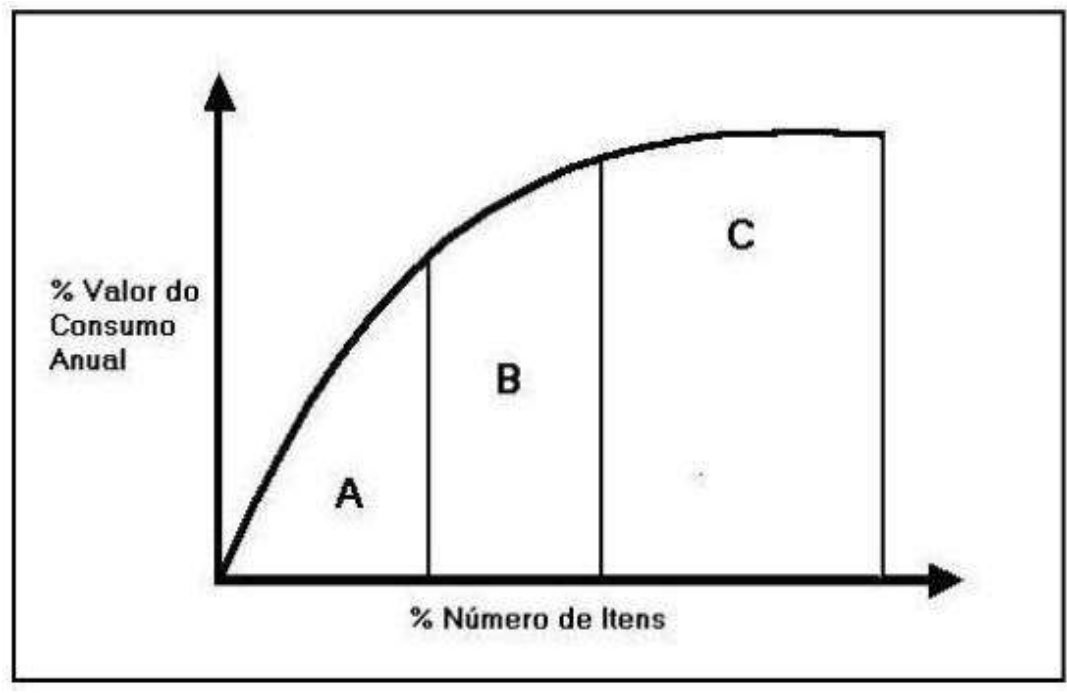

Figura 1: Curva ABC

Desse modo a Curva ABC tem como objetivo direcionar os esforços de melhoria, identificando os ítens mais importantes em termos de quantidade de ocorrências ou de impacto dos custos.

\subsubsection{Matriz GUT}

Segundo Carvalho e Almeida (2017), essa é uma ferramenta utilizada para o gerenciamento de riscos quanto ao ordenamento das prioridades. Silva e Oliveira (2019), destacam que se trata de uma metodologia que está diretamente ligada à fase de planejamento do ciclo PDCA, complementando assim, outras ferramentas da qualidade. Sua elaboração leva em consideração a gravidade, urgência e tendência dos problemas estudados. A gravidade está relacionado com o impacto causado ao longo do tempo, já a tendência quanto a capacidade de crescimento, redução e aparecimento, e a urgência está ligada ao tempo necessário para resolver o problema. Assim, pode-se observar a Tabela 1, abaixo. 
Tabela 1 - Critérios para classificação

\begin{tabular}{|c|c|c|c|}
\hline PONTOS & GRAVIDADE & URGÊNCIA & TENDÊNCIA \\
\hline 5 & Extremamente grave & Ação imediata & $\begin{array}{c}\text { Tende a piorar de } \\
\text { imediato }\end{array}$ \\
\hline 4 & Muito grave & $\begin{array}{c}\text { Com alguma } \\
\text { urgência }\end{array}$ & $\begin{array}{c}\text { Vai piorar a curto } \\
\text { prazo }\end{array}$ \\
\hline 3 & Grave & O mais cedo possível & $\begin{array}{c}\text { Vai piorar a médio } \\
\text { prazo }\end{array}$ \\
\hline 2 & Pouco grave & $\begin{array}{c}\text { Pode esperar um } \\
\text { pouco }\end{array}$ & $\begin{array}{c}\text { Vai piorar a longo } \\
\text { prazo }\end{array}$ \\
\hline 1 & Sem gravidade & Não tem pressa & Não vai piorar \\
\hline
\end{tabular}

Observa-se que para cada um dos critérios é atribuido uma nota que varia em uma escala de 1 á 5 e a importancia total de cada risco ocorre pela multiplicação dos fatores de gravidade, urgência e tendência, gerando assim o valor GUT.

\subsubsection{Diagrama de ishikawa}

O diagrama de ishikawa é comumente conhecido como diagrama de causa e efeito ou espinha de peixe, devido sua forma. Para Rodrigues e Costa (2009), trata-se de uma ferramenta que representa graficamente as relações de causalidade de um problema, de modo qualitativo, levando em consideração alguns facilitadores para elaboração, sendo eles os 6M's: método, máquina, medida, meio ambiente, material e mão de obra. Werkema já em 1995, complementava que a sua elaboração poderia servir como norteador no processo de identificação das atividades de melhoria e/ou medidas corretivas. As possíveis causas para os problemas estudados poderão ser listadas através de um brainstorming, ou seja, uma tempestade de ideias. Diante do exposto, Fornari Júnior (2010) afirma que o processo de elaboração do diagrama se dar por meio de algumas etapas, na qual destaca-se a definição do problema, identificação das possíveis causas e determinação de correlações existentes.

\subsection{Método Delphi}

O método Delphi, trata-se de uma ferramenta metodológica que pode ser aplicada em diferentes áreas de pesquisa, permitindo reunir opiniões de diferentes especialistas. Marques e Freitas (2018) explicam que o procedimento se dar através da aplicação de um questionário a diversos profissionais que deverão respondê-lo individualmente. Logo, o pesquisador assume a figura de moderador, recolhendo os resultados e observando as opiniões e justificativas para repassar ao grupo, no qual, após analisar os diversos pontos de vista, poderão alterar suas respostas. Destaca-se que esse processo deverá acontecer até que se consiga chegar em um consenso para melhor representatividade das informações.

\section{METODOLOGIA}

O presente trabalho se dividiu em três etapas, sendo elas: levantamento bibliográfico, pesquisa de campo e análise dos dados, fazendo uso das ferramentas da qualidade. Nesta primeira fase, buscou-se aprimorar os conhecimentos adquirindo um embasamento teórico através de livros e mídias digitais, a fim de facilitar o desenvolvimento do trabalho em questão, a saber: manifestações patológicas nas construções, ferramentas da qualidade e o método Delphi.

Por seguinte, a pesquisa se caracterizou como um estudo de campo que, segundo Gil (2002), trata-se da análise de uma comunidade através das técnicas de observação e entrevistas, podendo ser complementado com a análise de filmagens, documentos e fotografias. Nesse segundo momento, escolheram-se os bairros seguindo o critério de propensão para moradia, sendo eles: Centro, Alto São Francisco, Baviera e Lagoa, localizados na cidade de Quixadá - CE. Em seguida, realizou-se visitas in loco as edificações residênciais. Buscou-se, identificar as manifestações patológicas por meio de observação e registrá-las fotograficamente, sempre com autorização formal dos moradores. Eles assinaram um termo permitindo o uso dos dados para fins de pesquisa.

Já na terceira e última fase, analisaram-se os dados aplicando as ferramentas da qualidade com base nos registros fotográficos. Desta forma, elaborou-se a Folha de Verificação, fazendo uso da estratificação por bairro, edificação visitada 
e tipo de manifestação patológica. Depois, construiu-se a Curva ABC para diagnóstico das anomalias mais recorrentes em termos quantitativos. Por diante, utilizou-se a Matriz GUT, para priorização de solução das manifestações patológicas identificadas e estudadas, utilizando a técnica de opinião especializada com auxílio do método Delphi, útil na tomada de decisões. Por fim, construiu-se o Diagrama de Ishikawa para relacionar as possíveis causas e propôs-se medidas preventivas para a anomalia que compromete com maior intensidade as edificações.

\section{RESULTADOS E DISCUSSÕES}

Realizou-se visitas in loco a edificações de diferentes bairros localizados na cidade de Quixadá-CE. O tamanho total da amostra corresponde a 55 edificações residenciais, sendo que, 10 pertencem ao bairro Lagoa e outras 15 para cada um dos bairros Centro, Alto São Francisco e Baviera. Quanto as manifestações patológicas encontradas registraram-se um total de 167, correspondendo ao número de ocorrências das patologias (categoria) nos imóveis. No decorrer dessas visitas, regitrou-se fotograficamente as manifestações patológicas identificadas por meio da observação, como mostra o Quadro 1 a seguir, com algumas dessas anomalias.

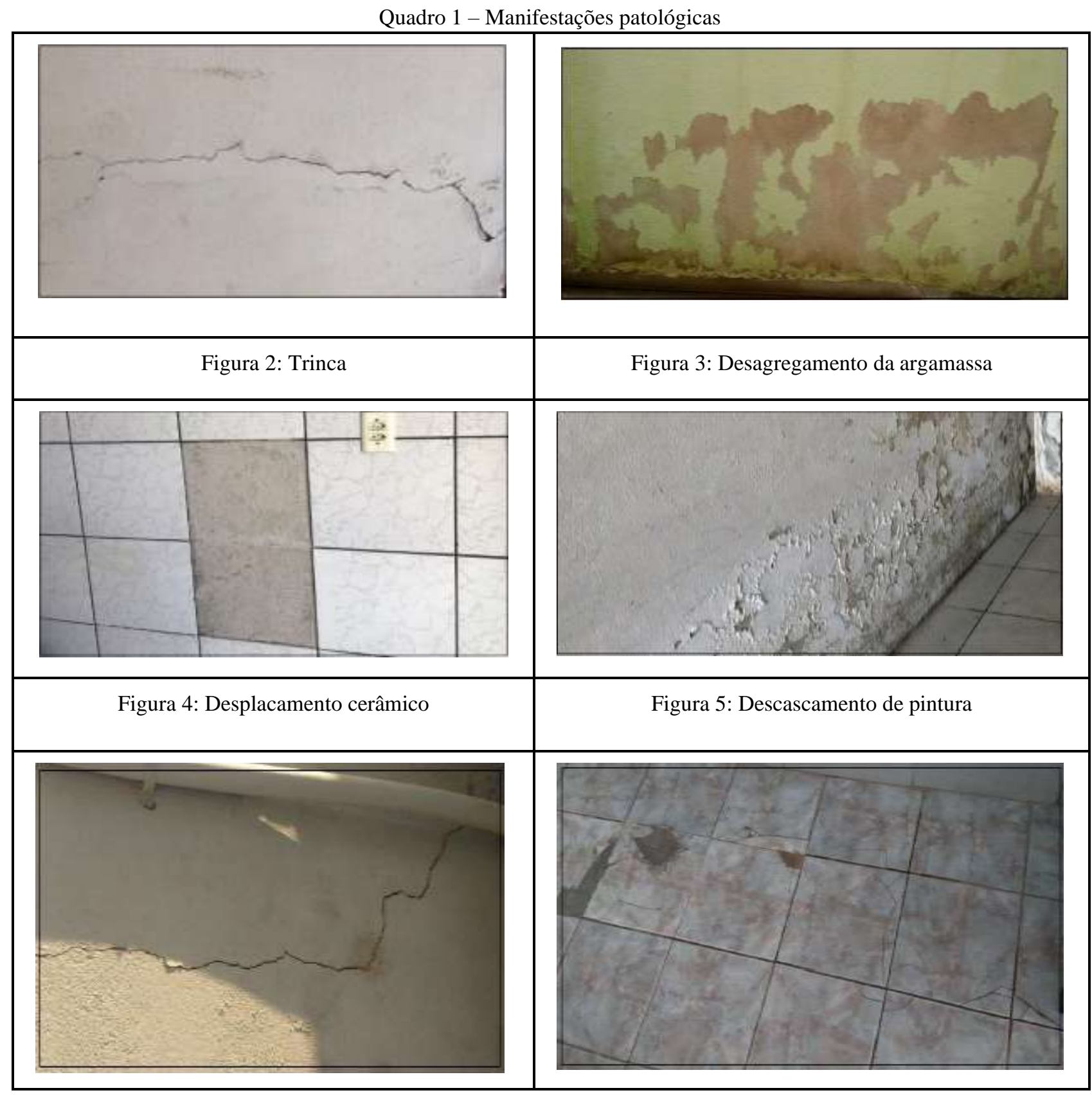




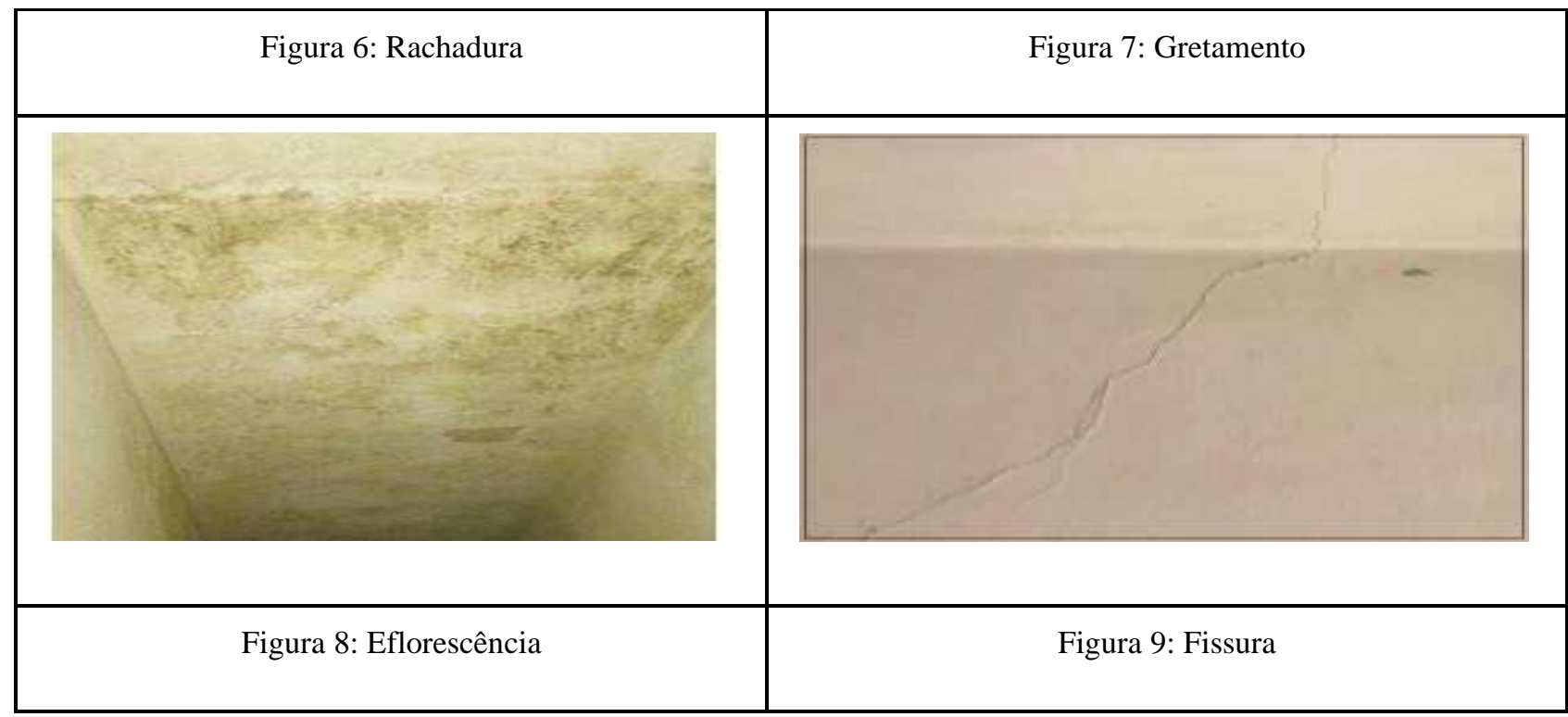

A partir de então, aplicaram-se as ferramentas da qualidade para coleta, disposição e tratamento dos dados. Em um primeiro momento, elaborou-se a folha de verificação realizando um levantamento quali-quantitativo dos problemas patológicos observados por meio dos registros fotográficos. Estratificou-a por bairro, edificação visitada e tipo de manifestação patológica. As anomalias observadas totalizaram-se em 10 tipos: fissuras, trincas, rachaduras, bolor, descascamento de pintura, eflorescência e desagregamento da argamassa, assim como os problemas nas placas cerâmicas, sendo eles: gretamento, deslocamentos e mudança de tonalidade. Os dados apresentam-se dispostos no Anexo 1.

Em seguida, visando a representação das informações de forma simples e organizada, facilitando a compreensão acerca dos resultados, elaborou-se a curva ABC com intuito de identificar as anomalias mais evidentes em termos quantitativos. Para a sua construção, estipulou-se os percentuais correspondentes à 60\%, 25\% e 15\% para caracterização dessas manifestações patológicas nas classes A, B e C, respectivamente. A Figura 10 a seguir, mostra a classificação desses problemas.

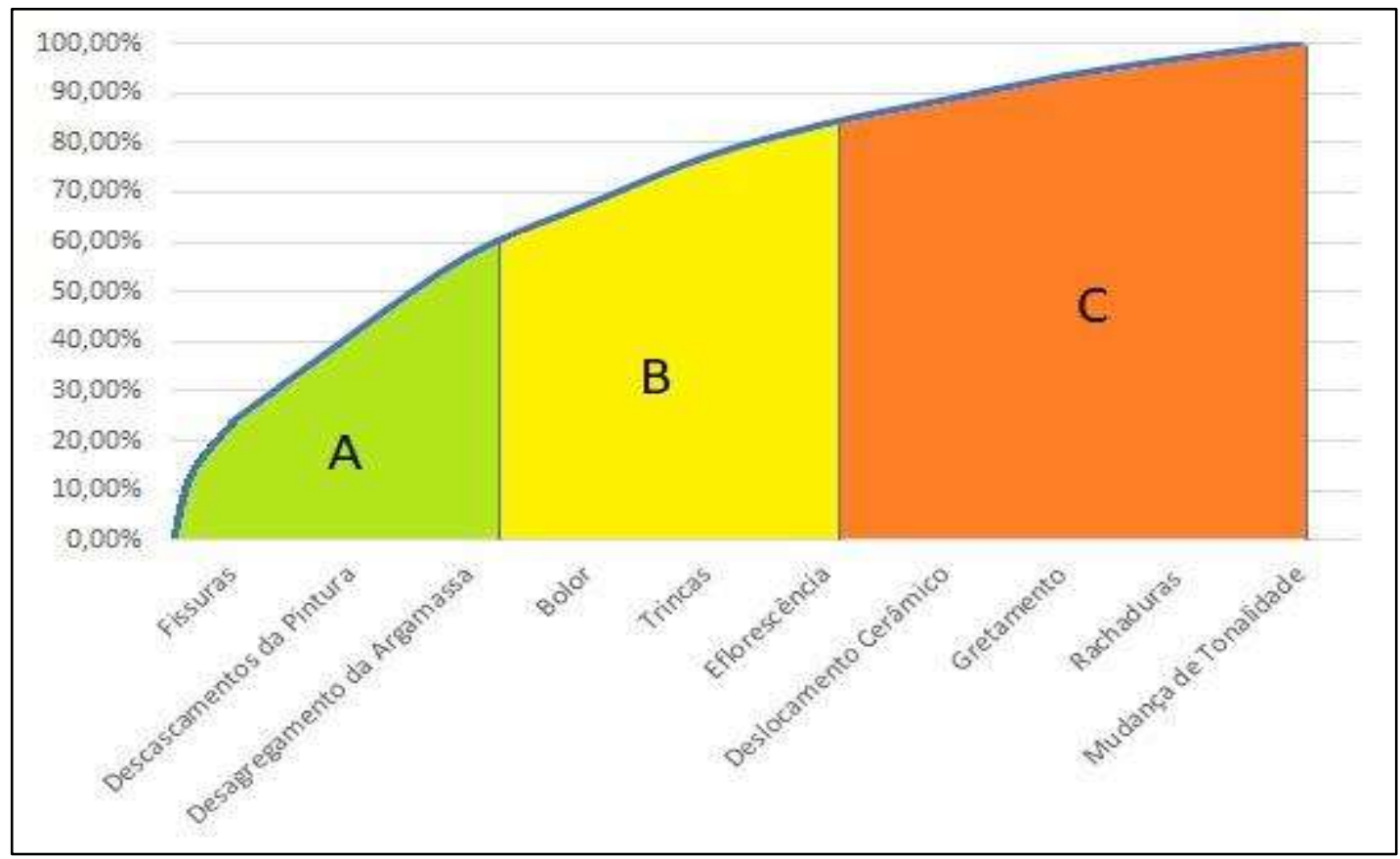

Figura 10: Curva ABC

Observa-se, que aproximadamente $60 \%$ das anomalias encontradas são referentes a apenas três manifestações patológicas: fissuras, descascamentos de pintura e desagregamento da argamassa. Desta forma, com apenas esses dados, poderia-se 
presumir que ao adotar medidas corretivas, mais da metade das manifestações patológicas observadas nos bairros Centro, Alto São Francisco, Lagoa e Baviera seriam solucionados. Porém, os problemas mais evidentes nem sempre apresentarão priorização para solução, uma vez que podem apresentar pouco risco a uma edificação, enquanto que outros menos recorrentes podem comprometer com maior intensidade a funcionalidade e segurança da moradia.

Diante disso, aplicou-se a matriz GUT: Gravidade, Urgência e Tendência para a classificação das anomalias identificadas e analisadas no estudo, priorizando-as de acordo com os parâmetros descritos no subtópico 2.2.3. No decorrer do processo de aplicação da ferramenta utilizou-se dos conceitos do método Delphi, a fim de melhorar e facilitar a tomada de decisões a partir das pontuações atribuídas pelos especialistas, sendo eles, dois Engenheiros Civis e um outro Tecnólogo em Construção de Edifícios. Nessa fase, realizou-se duas interações, até que se conseguisse entender a lógica e entrar em um concenso para atribuição das pontuações. Essas interações, foram realizadas através do intermédio dos autores que assumiram o papel de moderadores, compartilhando entre os profissionais as opiniões um dos outros. A Tabela 3, mostra as pontuações estabelecidas no valor GUT (G x U x T), obtido pela multiplicação entre as pontuações adotadas para a Gravidade, Urgência e Tendência em cada variável (manifestação patológica) e por cada um dos profissionais já na última interação.

Tabela 3 - Resultados da matriz GUT

\begin{tabular}{|c|c|c|c|}
\hline \multirow[b]{2}{*}{$\begin{array}{l}\text { MANIFESTACÓES } \\
\text { PATOLÓGICAS }\end{array}$} & \multicolumn{3}{|c|}{ RESULTADO GUT (G x U $x$ T) POR ESPECIALISTA } \\
\hline & Engenheiro Civil & Fngenheiro Civil & $\begin{array}{c}\text { Tecnólogo em Contrução de } \\
\text { Edificios }\end{array}$ \\
\hline Rachaduras & 50 & 80 & 60 \\
\hline Trincas & 45 & 48 & 25 \\
\hline Desagregamento da Argamassa & 24 & 18 & 6 \\
\hline Bolor & 6 & 18 & 2 \\
\hline Deslocamento Cerimico & 12 & 27 & 20 \\
\hline Gretamento & 12 & 4 & 4 \\
\hline Descascamentos da Pintura & 9 & 6 & 4 \\
\hline Fissuras & 27 & 12 & 8 \\
\hline Eflorescência & 2 & 18 & 12 \\
\hline Mudança de Tonalidade (Cerrimica) & 2 & 2 & 1 \\
\hline
\end{tabular}

As manifestações patológicas podem apresentar comportamentos distintos em diferentes situações, todavia, a análise realizada trata-se de uma percepção geral dos fatos. Com as pontuações estabelecidas na matriz GUT: Gravidade, Urgência e Tendência, percebe-se que para os Engenheiros Civis, assim como o Tecnólogo em Contrução de Edifícios as rachaduras necessitam de maior atenção, visto que compromete o sistema estrutural com maior intensidade. Diante disso, foram investigadas e apontadas por meio de um brainstorming entre os autores, as possíveis causas para as rachaduras nas edificações no decorrer do processo construtivo conforme a Figura 11 e durante a fase de utilização das edificações como mostra a Figura 12.

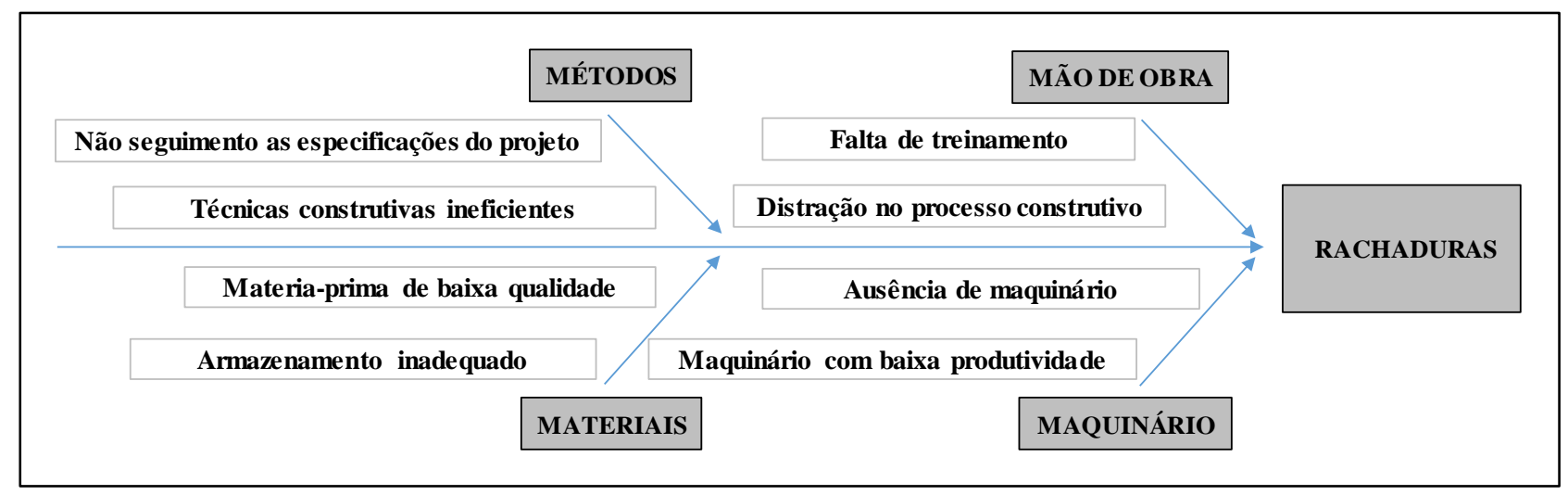

Figura 11: Diagrama de causa e efeito no processo de execução

$\mathrm{Na}$ Figura 11, foi especificado as possíveis causas de origem endógena para as rachaduras, em outras palavras, para aquelas provenientes de falhas no processo construtivo, especificamente na fase de execução. As causas primárias são: mão de obra, métodos, materiais e maquinário, e para cada uma dessas tem-se as causas secundárias que vão desde a ausência de capacitação da mão de obra, que influencia diretamente na execução adequada dos serviços, utilização 
inadequada dos materiais que podem comprometer o desempenho da estrutura, assim como metodos e equipamentos obsoletos.

Já na Figura 12, foram apontadas as causas para as anomalias congênitas, executiva, adquirida e acidental, sendo essas devido a erros no projeto, execução, utilização e fatores naturais ou acidentes, respectivamente.

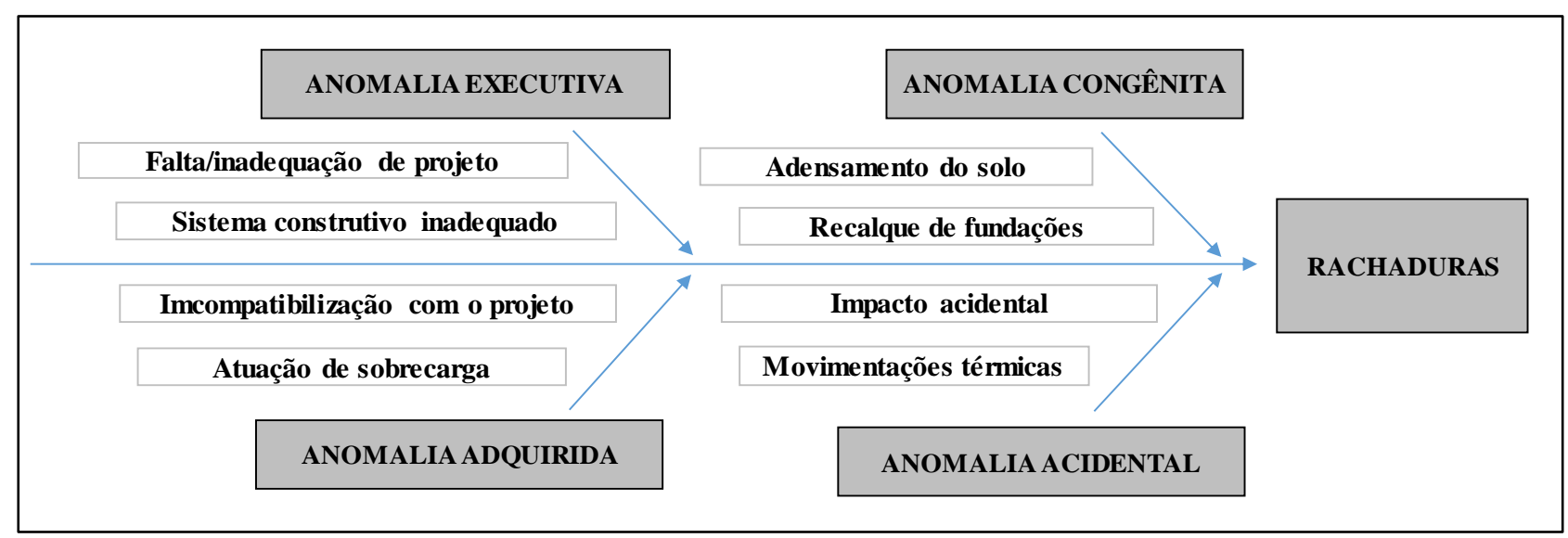

Figura 12: Diagrama de causa e efeito no processo construtivo

Nesse caso, observa-se que a inadequação de projetos em casos de ampliação e reforma, atuação de sobrecarga na estrutura além do especificado, adensamentos do solo e movimentações térmicas são fatores que influenciam no surgimento de rachaduras nas edificações, principalmente quando não se faz manutenções periódicas.

\section{CONCLUSÕES}

De acordo com o estudo realizado, pôde-se ampliar os conhecimentos acerca da aplicabilidade das ferramentas da qualidade para análise das manifestações patológicas observadas em edificações residênciais. Diagnosticou-se que, as anomalias evidentes foram: fissuras, trincas, rachaduras, bolor, descascamento de pintura, eflorescência, desagregamento da argamassa, assim como gretamento, deslocamento das placas cerâmicas e mudança de tonalidade das mesmas. Constatatou-se por meio da Curva $\mathrm{ABC}$ que as fissuras, descascamentos de pintura e desagregamentos da argamassa são as anomalias mais evidentes em termos quantitativos nos bairros Centro, Alto São Francisco, Baviera e Lagoa em Quixadá-CE. Diante disso, apontou-se atravez da Matriz GUT: Gravidade, Urgência e Tendência e com auxílio do Método Delphi a ordem para priorização de solução dos problemas estudados e analisados e identificou-se que as rachaduras são, aparentemente, as anomalias que comprometem com maior intensidade a funcionalidade e segurança das moradias.

Dado o exposto, apontou-se por meio do Diagrama de Ishikawa as possíveis causas e propôs-se medidas mitigatórias, para a manifestação patológica que segundo alguns especialistas apresenta maior risco a edificação, no caso as rachaduras. Assim, para as novas construções indica-se realizar o estudo geotécnico do solo, desenvolver projetos bem dimensionados prezando pela compatibilização dos mesmos, utilizar materiais com qualidade e executar os serviços com mão de obra especializada. No decorrer do tempo de utilização da edificação deve-se realizar manutenções frequentemente.

Dado o exposto, constata-se que o presente trabalho apresenta uma contribuição no âmbito social e educacional, disponibilizando dados capazes de nortear os contrutores quanto a necessidade de adotar-se medidas preventivas no decorrer do processo construvivo, capazes de evitar o surgimento das anomalias evidenciadas no presente trabalho. Além disso, o estudo em questão abre a vertente para o desenvolvimento de novos trabalhos acadêmicos nessa linha de pesquisa.

\section{REFERÊNCIAS}

CARVALHO. E.M. ALMEIDA. L.S. Check-list para inspeções prediais residenciais de múltiplos pavimentos: desenvolvimento e aplicação. Foz do Iuaçu - PR. 2017.

FORNARI JUNIOR. C. C. M. Aplicação da ferramenta da qualidade (Diagrama de Ishikawa) e do PDCA no desenvolvimento de pesquisa para a reutilização dos resíduos sólidos de coco verde. Ingepro. 2010. 
FIGUEIREDO. D. L. M. LAPA. J. S. Patologia, recuperação e reparo das estruturas de concreto. Belo Horizonte. 2008.

GIL.A.C. Como elaborar projetos de pesquisa. 4 ed. São Paulo. Atlas. 2002.

MARQUES. J.B.V. FREITAS. D. Método DELPHI: caracterização e potencialidades na pesquisa em educação. Pro posições. V.29, N. 2. 2018.

MARSHALL JÚNIOR, Isnard et al. Gestão da Qualidade. 9. Ed. - Rio de Janeiro: Editora FGV, 2008

MOREIRA. D. C. MARCHINI. N. R. Estudo de caso das manifestações patológicas em uma residência térrea na cidade de Paiçandu - PR. Maringá - PR. 2018.

NOBRE JÚMIOR. J.C.R. MELO. S.B. MATOS. P.R.F. Alterações no gerenciamento de estoque de uma microempresa do setor têxtil através da aplicação da cirva ABC e outras ferramentas: estudo de caso em Caucaia CE. ENEGEP. Santos - SP. 2019

RODRIGUES. M.F.O. COSTA. J.M. Análise de risco em projectos de construção. Portugal. 2009.

SILVA. A.R.M. OLIVEIRA. A.D. Avaliação patológica na central de aulas IV da UFERSA campus Mossoró de acordo com a metodologia da matriz GUT. Mossoró. 2019.

SOLANO. R.S. PALADINI. E.P. Curva ABC de fornecedores: uma contribuição ao planejamento, programação, controle e gerenciamento de empreendimentos e obras. Florianópolis. 2003.

SOUZA, V. C. M.; RIPPER, T. Patologia, recuperação e reforço de estruturas de concreto. São Paulo, Editora Pini. 1998.

SANTOS. C. F. SOARES. J. M. D. Patologia de estrutura de concreto armado. Santa Maria - RS. 2014.

VIEIRA. M. A. Patologias construtivas: conceito, origens e método de tratamento. Especialize. Uberlândia - MG. 2016.

WERKEMA. M.C.C. Ferramentas estatísticas básicas para o gerenciamento de processos. Belo Horizonte - MG. 1995. 
ANEXO 1 - FOLHA DE VERIFICAÇÃO

\begin{tabular}{|c|c|c|c|c|c|c|c|c|c|c|c|}
\hline \multirow[t]{3}{*}{ BA IRROS } & \multirow[t]{3}{*}{ EDIFICAÇÃo } & \multicolumn{10}{|c|}{ PROBLEMAS PA TOLÓGICOS } \\
\hline & & & Fissura: & & & Problem as de $P$ & intura & $\begin{array}{l}\text { Proble m as de } \\
\text { Revestim ento }\end{array}$ & Probl & m as Cerâmic & \\
\hline & & Fissuras & Trinas & Fachaduras & Bobr & Descascamertos & Efbrescênnda & $\begin{array}{l}\text { Desagregament o da } \\
\text { argmassa }\end{array}$ & Desbamertos & $\begin{array}{l}\text { Mudarya de } \\
\text { Tonalidade }\end{array}$ & Gretamerto \\
\hline \multirow{15}{*}{ 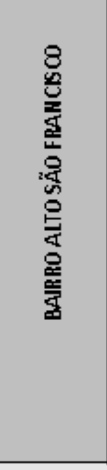 } & Casa 1 & $\mathrm{x}$ & & & $x$ & $x$ & & $\mathrm{x}$ & & & \\
\hline & Casa 2 & $x$ & & & & & & & $x$ & & \\
\hline & Casa 3 & $\mathrm{x}$ & & & & $\mathrm{x}$ & & $x$ & & & \\
\hline & Casa 4 & $\mathrm{x}$ & $\mathrm{x}$ & & $\mathrm{x}$ & $\mathrm{x}$ & & & & & \\
\hline & Casa 5 & $\mathrm{x}$ & $\mathrm{x}$ & & & & $\mathrm{x}$ & & $\mathrm{x}$ & & \\
\hline & Casa 6 & $x$ & & & & & & & & & \\
\hline & Casa 7 & & $\mathrm{x}$ & & & $\mathrm{x}$ & & & $\mathrm{x}$ & & \\
\hline & Casa 8 & $\mathrm{x}$ & & & & $\mathrm{x}$ & & & & & \\
\hline & Casa 9 & $\mathrm{x}$ & & & $\mathrm{x}$ & & & & & & \\
\hline & Casa10 & $\mathrm{x}$ & & & & & & $\mathrm{x}$ & & & \\
\hline & Casa11 & $x$ & & & & $\mathrm{x}$ & & $x$ & & & \\
\hline & Casa12 & $\mathrm{x}$ & & & & $\mathrm{x}$ & & & & & \\
\hline & Casa13 & $\mathrm{x}$ & & & & & & $\mathrm{x}$ & & & \\
\hline & Casa14 & & $\mathrm{x}$ & & $\mathrm{x}$ & & & $\mathrm{x}$ & & & \\
\hline & Casa15 & $x$ & & & $x$ & & & $x$ & & & \\
\hline & & & & & & & & & & & \\
\hline \multirow{15}{*}{ 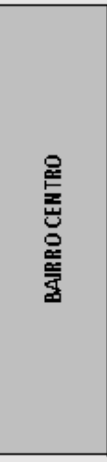 } & Casa 1 & $x$ & & & & & $\mathrm{x}$ & & & & \\
\hline & Casa 2 & $\mathrm{x}$ & & & & $\mathrm{x}$ & & & & & \\
\hline & Casa 3 & $x$ & & & & & & & & & \\
\hline & Casa 4 & $\mathrm{x}$ & & & & & & & & & \\
\hline & Casa 5 & $x$ & & & $x$ & & $x$ & & & & \\
\hline & Casa 6 & $\mathrm{x}$ & & & & & & & & & \\
\hline & Casa 7 & & & & $x$ & $\mathrm{x}$ & & $\mathrm{x}$ & & & \\
\hline & Casa 8 & _ & & & & & & & & & $\mathrm{x}$ \\
\hline & Casa 9 & & & $x$ & & $\mathrm{x}$ & & & & & \\
\hline & Casa10 & & & & $\mathrm{x}$ & & & & & & \\
\hline & Casa11 & $\mathrm{x}$ & & & & & $\mathrm{x}$ & & & & \\
\hline & Casa12 & $\mathrm{x}$ & & $x$ & $x$ & $\mathrm{x}$ & $x$ & $x$ & & & \\
\hline & Casa13 & $\mathrm{x}$ & $x$ & & & & & & & & \\
\hline & Casa14 & $x$ & & & & & & & & & \\
\hline & Casa15 & $x$ & & & & & & & & & \\
\hline \multirow{15}{*}{ 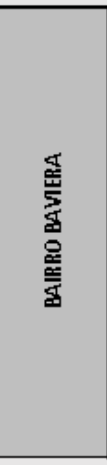 } & Case1 & $x$ & $x$ & $x$ & $x$ & $x$ & $x$ & $x$ & 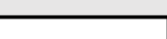 & & \\
\hline & Casa 2 & $\mathrm{x}$ & $\mathrm{x}$ & $n$ & $n$ & $x$ & $x^{2}$ & $n^{\prime}$ & & & \\
\hline & Casa 3 & & $\mathrm{x}$ & & & $\mathrm{x}$ & $\mathrm{x}$ & $\mathrm{x}$ & $\mathrm{x}$ & & \\
\hline & Casa 4 & $\mathrm{x}$ & $\mathrm{x}$ & & & $x$ & & $x$ & & & \\
\hline & Casa 5 & & & & $x$ & $x$ & & & & $\mathrm{x}$ & $\mathrm{x}$ \\
\hline & Casa 6 & $x$ & $\mathrm{x}$ & & & $\mathrm{x}$ & & $\mathrm{x}$ & & - & \\
\hline & Casa 7 & $\mathrm{x}$ & $x$ & & & $x$ & & $x$ & & & \\
\hline & Casa 8 & $\mathrm{x}$ & $\mathrm{x}$ & & $x$ & & & & & & $x$ \\
\hline & Casa 9 & $\mathrm{x}$ & & $x$ & $x$ & & & $x$ & & $\mathrm{x}$ & \\
\hline & Casa10 & $x$ & & & & $x$ & & & $\mathrm{x}$ & & \\
\hline & Casa11 & $x$ & $x$ & $\mathrm{x}$ & & & & $\mathrm{x}$ & & & \\
\hline & Casa12 & $x$ & $x$ & & & $x$ & & $\mathrm{x}$ & & & \\
\hline & Casa13 & $\mathrm{x}$ & & $x$ & $x$ & $\mathrm{x}$ & $x$ & & & & \\
\hline & Casa14 & $x$ & $x$ & & & $x$ & $x$ & $x$ & $x$ & $x$ & $x$ \\
\hline & Casa15 & & $x$ & & & $x$ & & $x$ & & $x$ & $x$ \\
\hline \multirow{10}{*}{ 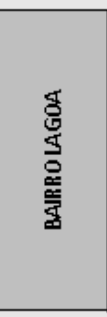 } & Casa 1 & & & & 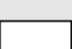 & & & & & & \\
\hline & $\begin{array}{l}\text { Casa } 1 \\
\text { Casa } 2 \\
\end{array}$ & $\frac{x}{x}$ & & & $x$ & $x$ & & $\frac{x}{x}$ & & & $x$ \\
\hline & Casa 3 & & & & & $\mathrm{x}$ & $x$ & $x$ & & $x$ & $x$ \\
\hline & Casa 4 & $x$ & & & $x$ & & & $x$ & & & \\
\hline & Casa 5 & $x$ & & & & & & $x$ & $x$ & & \\
\hline & Casa 6 & & & & & $x$ & & & & & \\
\hline & Casa 7 & & & & & $x$ & & & & & \\
\hline & Casa 8 & & & & & $\mathrm{x}$ & & $x$ & & & \\
\hline & Casa 9 & & & & $x$ & $\mathrm{x}$ & $x$ & $x$ & & & \\
\hline & Casa10 & & & & & & & $x$ & $x$ & & $x$ \\
\hline \multirow{2}{*}{\multicolumn{2}{|c|}{ TO TAL }} & 40 & 16 & 6 & 17 & 29 & 11 & 27 & 8 & 5 & 8 \\
\hline & & & 62 & & & 57 & & 27 & & 21 & \\
\hline
\end{tabular}

